\title{
Ex Vivo Produced Oral Mucosa Equivalent by Using the Direct Explant Cell Culture Technique
}

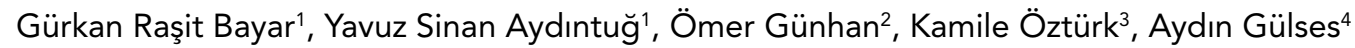 \\ ${ }^{1}$ Department of Oral and Maxillofacial Surgery, Gülhane Military Medical Academy, Ankara, Turkey \\ 2Department of Pathology, Gülhane Military Medical Academy, Ankara, Turkey \\ ${ }^{3}$ Department of Biology, Faculty of Medicine, Hacettepe University, Ankara, Turkey \\ ${ }^{4}$ Surgical Infirmary, $2^{\text {nd }}$ Army Corps, Commando Troop No:5, Gökçeada, Çanakkale, Turkey
}

\begin{abstract}
Objective: The aim of this study is the histological and immunohistochemical evaluation of ex vivo produced oral mucosal equivalents using keratinocytes cultured by direct explant technique.

Material and Methods: Oral mucosa tissue samples were obtained from the keratinized gingival tissues of 14 healthy human subjects. Human oral mucosa keratinocytes from an oral mucosa biopsy specimen were dissociated by the explant technique. Once a sufficient population of keratinocytes was reached, they were seeded onto the type IV collagen coated "AlloDerm" and taken for histological and immunohistochemical examinations at 11 days postseeding of the keratinocytes on the cadaveric human dermal matrix.
\end{abstract}

Results: Histopathologically and immunohistochemically, 12 out of 14 successful ex vivo produced oral mucosa equivalents (EVPOME) that consisted of a stratified epidermis on a dermal matrix have been developed with keratinocytes cultured by the explant technique.

Conclusion: The technical handling involved in the direct explant method at the beginning of the process has fewer steps than the enzymatic method and use of the direct explant technique protocol for culturing of human oral mucosa keratinocyte may be more adequate for EVPOME production.

Key Words: Oral mucosa, keratinocyte, epidermis, dermal matrix

Received: $18.10 .2011 \quad$ Accepted: 01.02.2012

\section{Introduction}

Burns, traumas, penetrating gun shot injuries and ablative cancer surgeries of the circumoral region could result in composite tissue defects including the oral mucosa. There are many techniques described in the literature aiming at the reconstruction of the intraoral region including: the use of skinbearing flaps, full thickness mucosal grafts and split thickness skin grafts, grafting procedures by using allogenic materials, the use of soft tissue expanders, etc (1-5). However, inadequate texture of the skin grafts due to the unwanted hair growth and excessive keratinization, insufficient volume of the full thickness mucosal grafts, donor site morbidity and the controversies in reported success rates of the soft tissue expanders and allogenic graft materials resulted in the need for tissue engineering techniques which may provide new alternatives to overcome these problems. In the last two decades, oral mucosa equivalents produced in ex-vivo conditions began to assist to oral and maxillofacial surgeons in the management of the oral mucosa deficiencies. In addition, researches in the fields of oral biology, pharmacology, toxicology and chromosomal analyses are widely performed by using the "ex-vivo produced oral mucosa equivalents" ("EVPOME").

The production of an oral mucosa equivalent consists of two steps: Keratinocyte cultivation (primary cell culture) and the production of the oral mucosa equivalent on a scaffold. Basically, there are two techniques used in cultivation of oral mucosa keratinocytes: the enzymatic and the direct explant technique $(6,7)$.

Billingham and Reynolds (8) proposed a technique for the separation of epithelial cells using an enzyme (trypsin), thus called the enzymatic method, in order to obtain keratinocytes and at the same time prevent these cells from losing their viability and culture potential. In 1999, Izumi et al. (9) developed an "ex vivo composite oral mucosal equivalent" that consisted of stratified epidermis on an acellular nonimmunogenic cadaveric human dermis matrix, and which has become widespread via successful clinical human intraoral grafting procedures during the last decade (10-12). However, several technical obstacles in the enzymatic method can be encountered, such as long culture periods (3-4 weeks) and low intake rates in the enzymatic treatment $(13,14)$. 
In 1910, Carrel and Burrows described a method for the extraction of epithelial cells called direct explant, which has been used since that time (15). The direct explant technique has also been used for 30 years in the culturing of human oral tissues $(16,17)$. In the direct explant technique, the cells from the initial tissue extraction affixed to a culture dish migrate out of the tissue starting from the edges of the fragment, adhering to and multiplying on the culture dish. It has been suggested that direct explant technique is more successful than the enzymatic technique in culturing human oral keratinocytes $(6,18)$.

In the current study, an optimized version of the original direct explant technique described by Carrel and Burrows in 1910 was used to obtain keratinocyte culture and produce "EVPOME". To the best of our knowledge, "EVPOME" was first produced by using the direct explant technique.

\section{Material and Methods}

This project was approved by the First Ethics Committee of Clinical Researches of Ankara, under License Number 2010/01-214. Primary cell cultures were performed by using human oral epithelial tissues from volunteers undergoing dental surgeries such as; implant surgery, third molar extraction, and gum surgery etc. at the Oral and Maxillofacial Surgery Clinics in Gulhane Military Medical Academy. Oral epithelial tissues were obtained from the keratinized gingival tissues of 14 healthy human subjects ( 8 male, 6 female and age ranging from 16 to 57 years).

\section{Primary culture $(19,20)$}

The tissue specimens were carried to the cell culture laboratory in a $10 \mathrm{~mL}$. "culture media" ("Dulbecco's Modified Eagle's Medium" DMEM: Gibco BRL, New York, USA $\mathrm{pH}$ 7.2) supplemented with $10 \%$ heat-inactivated fetal calf serum (FCS), $100 \mathrm{U} / \mathrm{mL}$ penicillin, $100 \mu \mathrm{g} / \mathrm{mL}$ streptomycin and $0.5 \%$ amphotericin $B$ (Gibco BRL, New York, USA) to prevent growth of micro-organisms. The tissue specimens were washed and disinfected in a pure povidone iodine solution for 1-2 minutes and then washed in culture media. Then, each tissue specimen were cut into 8-10 pieces, approximately $1 \times 1 \mathrm{~mm}$ in size, and placed in the different culture flasks (T-25 flask, Corning, New York, USA) by a sterile needle of the dental injector (Figure 1). Tissue pieces were left in the culture plate for 15-20 minutes and then the culture media was dropped on the tissue pieces gently. After waiting of 3-4 hours, culture plate was flooded with $5 \mathrm{~mL}$. culture media. The culture plate was incubated at $37^{\circ} \mathrm{C}$ in a humidified atmosphere of $95 \%$ air and $5 \% \mathrm{CO}_{2}$ (Incubator SANYO MCO 18-AIC, Osaka, Japan). The culture medium was replaced with the fresh one twice a week. After the keratinocytes, which were squamous in shape, started to multiply around the tissue sample origin to a diameter of 2-5 mm (Figure 2a), the culture medium was changed to a chemically defined serum-free culture medium (Figure 2b) ("EpiLife" Cascade Biologics, Portland, OR, USA) supplemented with human keratinocyte growth factors ("EDGS" Cascade Biologics, Portland, OR, USA), $125 \mu \mathrm{g} / \mathrm{mL}$ gentamycin and $1 \mu \mathrm{g} /$ $\mathrm{mL}$ amphotericine B (Sigma Chemicals Co, USA) with a cal- cium concentration of $0.06 \mathrm{mM}$. Thus, fibroblast overgrowth was prevented (Figure 2c). The culture was fed every other day with the chemically defined serum-free culture medium. After around 20 days, when the primary cell culture reached $70-80 \%$ confluence (Figure $2 \mathrm{~d}$ ), oral mucosa keratinocytes were harvested with a solution of $0.025 \%$ trypsin-ethylenediaminetetra-acetic acid (Trypsin-EDTA, "TE" Cascade Biologics, Portland, OR, USA) at $37^{\circ} \mathrm{C}$. After 4-5 minutes, TrypsinEDTA activity was inhibited with an equal volume of $0.0125 \%$ trypsin inhibitor. Disaggregated cells were collected, counted, centrifuged, resuspended and replated into new T-25 flasks (Corning, New York, USA) at a density of $2.0 \times 10^{4}$ cells/

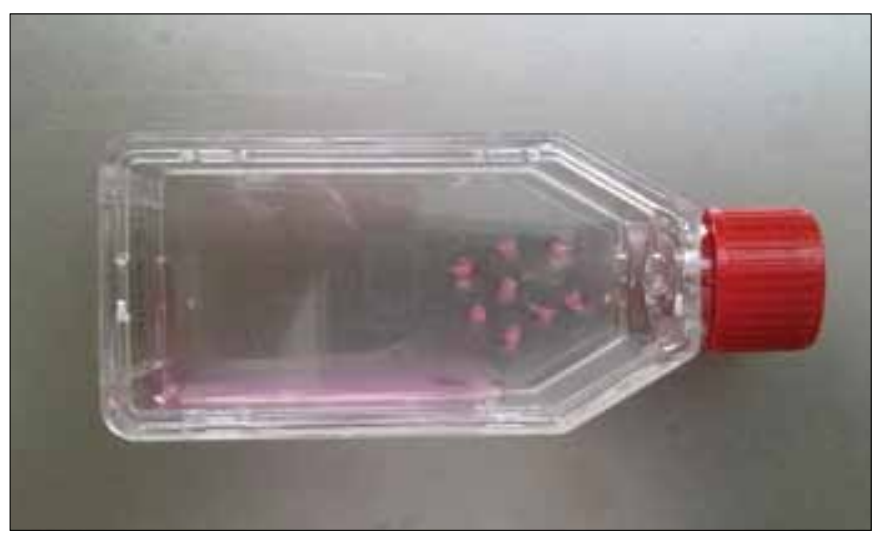

Figure 1. The tissue specimens, approximately $1 \times 1 \mathrm{~mm}$ in size, placed in a T-25 flask

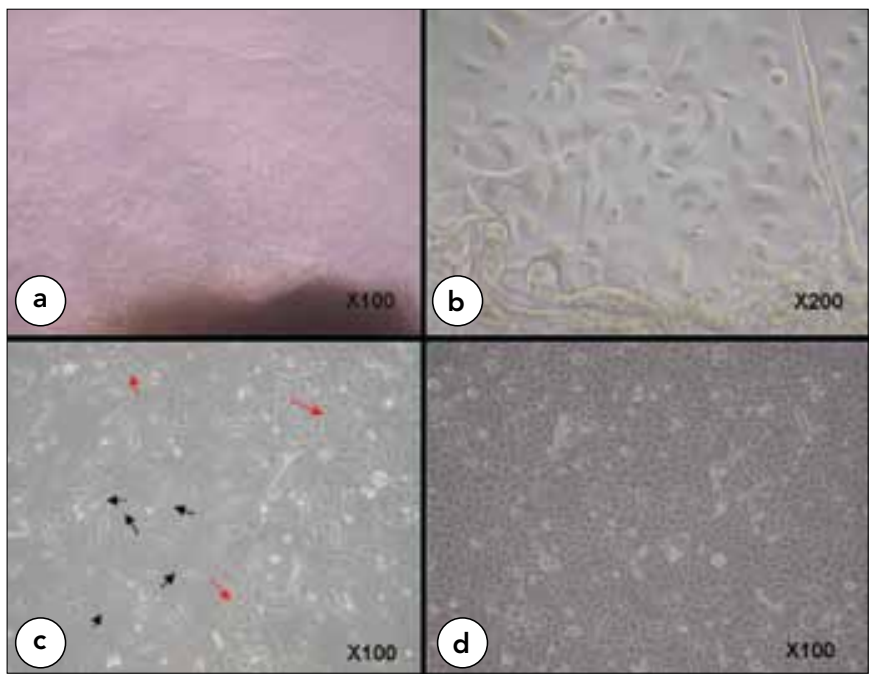

Figure 2. a) After the keratinocytes, which are squamous in shape, start to multiply around the tissue sample origin to a diameter of $2-5 \mathrm{~mm}, \mathrm{~b}$ ) the culture medium is changed to a chemically defined serum-free culture medium ("EpiLife") supplemented with human keratinocyte growth factors, $125 \mu \mathrm{g} / \mathrm{mL}$ gentamycin, $1 \mu \mathrm{g} / \mathrm{mL}$ amphotericine $B$ and with a calcium concentration of $0.06 \mathrm{mM} \mathrm{c}$ ) Prevention of the fibroblast overgrowth (keratinocytes are shown by red arrows and fibroblasts are showed by black arrows). d) Hyperproliferative state of keratinocytes in a chemically defined serum-free culture medium. The cuboidal keratinocytes are relatively uniform in size and morphology, and there is no evidence of fibroblast contamination (x100) 
$\mathrm{cm}^{2}$ (9). In this study, the success rate of the culturing method was defined as the ability of the cells to grow out from the tissue sample origin, become $70-80 \%$ confluent and to survive at least until the first passage.

\section{Preparation of "AlloDerm" (9)}

"AlloDerm" (LifeCell, Branchburg, NJ), the acellular human cadaver dermis, was cut into circular pieces of $11.3 \mathrm{~mm}$ diameter in order to conform to the area of a 48-well microplate (Corning Inc. Corning, NY, USA). Two circular samples were rehydrated in washing solution. The epidermal surface of the human cadaver dermis is rough and washing solution is easily drained off, on the other hand, dermal surface is shiny and washing solution is retained. Epidermal and dermal surfaces of the samples were identified and transferred to the 48-well microplate with epidermal surfaces up. They were presoaked and kept overnight at $4^{\circ} \mathrm{C}$ in $100 \mu \mathrm{L}$ of PBS and human Type IV Collagen $\left(5 \mu \mathrm{g} / \mathrm{cm}^{2}\right)$ to enhance attachment of seeded keratinocytes.

\section{Production of keratinocytes on "AlloDerm" (9)}

Oral keratinocytes from the first passage of actively dividing cells were used to seed onto "AlloDerm" samples. They were harvested by adding a solution of trypsin-ethylenediaminetetra-acetic acid (Trypsin-EDTA, TE Cascade Biologics, Portland, OR, USA) at $37^{\circ} \mathrm{C}$. Trypsin-EDTA activity was inhibited with an equal volume of $0.0125 \%$ trypsin inhibitor. Disaggregated cells were collected, counted, centrifuged, and re-suspended. Cells were seeded onto "AlloDerm" samples in 2 different 48-well microplates at the density of $1.8 \times 10^{5}$ cells. At that time, $1.2 \mathrm{~mL}$ of "culture medium" containing a high concentration of calcium $(1.2 \mathrm{mM})$ was gently added to 48-well microplates without disturbing the cells. The oral keratinocyte-"AlloDerm" composites were cultured and submerged for 4 days in the 48-well culture microplates. Composites were fed daily during this period with "culture medium". After incubating the composites in a submerged environment for 4 days, they were transferred to organotypic tissue culture flasks (Corning Inc. Corning, NY, USA). The medium was changed every other day for 7 more days. The organotypic tissue culture flasks allowed the composites to grow at an air-liquid interface. This culturing technique has been shown to encourage stratification of the epithelial layers $(9,21)$.

\section{Histological examination}

Prior to keratinocyte cultivation, a piece of original tissue sample was obtained to create a control group and used for comparison of the histological and immunohistochemical characteristics of the original tissue sample and "EVPOME" of the same patient. A piece of tissue sample was fixed in $10 \%$ formaldehyde. Then it was embedded in paraffin and cut to $5 \mu \mathrm{m}$ and stained with hematoxylin and eosin. "EVPOME"s were removed from the organotypic tissue culture flasks at the $11^{\text {th }}$ day post-seeding of the keratinocytes and fixed in $10 \%$ formaldehyde. The fixed "EVPOME"s were embedded in paraffin and cut to $5 \mu \mathrm{m}$ and stained with hematoxylin and eosin in the same manner.

\section{Immunohistochemical examination}

Anti-Ki-67, anti-laminin-5 and anti-cytoceratin-3 (K-3) primary antibodies were used for the immunohistochemical evaluation. The comparative evaluation was performed in the same manner as the histological evaluation.

Four-micron thick sections were prepared. For Immunohistochemistry, slides were placed in an oven at $56^{\circ} \mathrm{C}$ dry heat for $30 \mathrm{~min}$ for deparaffinization, then washed in alcohol and xylol solutions. Slides were placed in citrate buffer at $98^{\circ} \mathrm{C}$ for 5 min for antigen retrieval. Then they were washed in Phosphate Buffered Saline (PBS). For blocking the endogenous peroxidase, sections were incubated in $3 \% \mathrm{H}_{2} \mathrm{O}_{2}$ for $10 \mathrm{~min}$. Slides were placed in distilled water and PBS, then incubated with primary antibodies (Antikeratin 3/76 clone AE5 at a 1:200 dilution, Anti Ki-67 at a 1:100 dilution, and Anti Laminin-5 clone P3H9-2 at a 1:100 dilution mouse monoclonal antibodies, Millipore Billerica, MA USA) for 2 hours at $25^{\circ} \mathrm{C}$ and washed with PBS. Secondary biotin-conjugated antimouse immunoglobulin was used at a 1: 500 dilution, and a horseradish peroxidaseconjugated streptavidin solution was applied for $40 \mathrm{~min}$ and washed with PBS. Application of diaminobenzidine hydrochloride chromogen for $10 \mathrm{~min}$ and washing with tap water were carried out. Slides were counterstained with hematoxylin and rinsed in tap water and were mounted.

\section{Results}

Tissue samples were obtained from 14 healthy human subjects ( 8 males and 6 females) aged between 16-57 years (mean age $28.5 \pm 11.4$ ). The results showed that the only two primary cultures of the oral epithelial cells by direct explant technique failed, and the total success rate was $85.71 \%$. In this study, no contamination of micro-organisms in primary cell cultures was observed. The success rate of the "EVPOME" production following a successful keratinocyte cultivation was $100 \%$ (Table 1).

The average number of days required for epithelial cells to grow and migrate out from the tissue origin was around 9-10 days and it took a total of $20.25 \pm 1.05$ days for the cells to become fully confluent.

\section{Histological evaluation}

Histologically, "EVPOME"s development showed multilayered epithelium comprising basal, suprabasal, and parakeratinized layers. The epithelial architecture of "EVPOME"s resembled that of normal oral mucosa. Highly stratified

Table 1. The success and failure of the direct explant technique classified by sex and age

\begin{tabular}{|c|c|c|c|c|}
\hline & & Success & Failure & Total \\
\hline Number of cases & & $\begin{array}{c}12 \\
(85.7 \%)\end{array}$ & $\begin{array}{c}2 \\
(14.3 \%)\end{array}$ & $\begin{array}{c}14 \\
(100 \%)\end{array}$ \\
\hline Age (Means+SD) & & $26.5 \pm 8.6$ & $41 \pm 22.6$ & $28.5 \pm 11.4$ \\
\hline Range (years) & & $16-43$ & $25-57$ & $16-57$ \\
\hline \multirow[t]{2}{*}{ Sex } & Male & 6 & 2 & 8 \\
\hline & Female & 6 & . & 6 \\
\hline
\end{tabular}




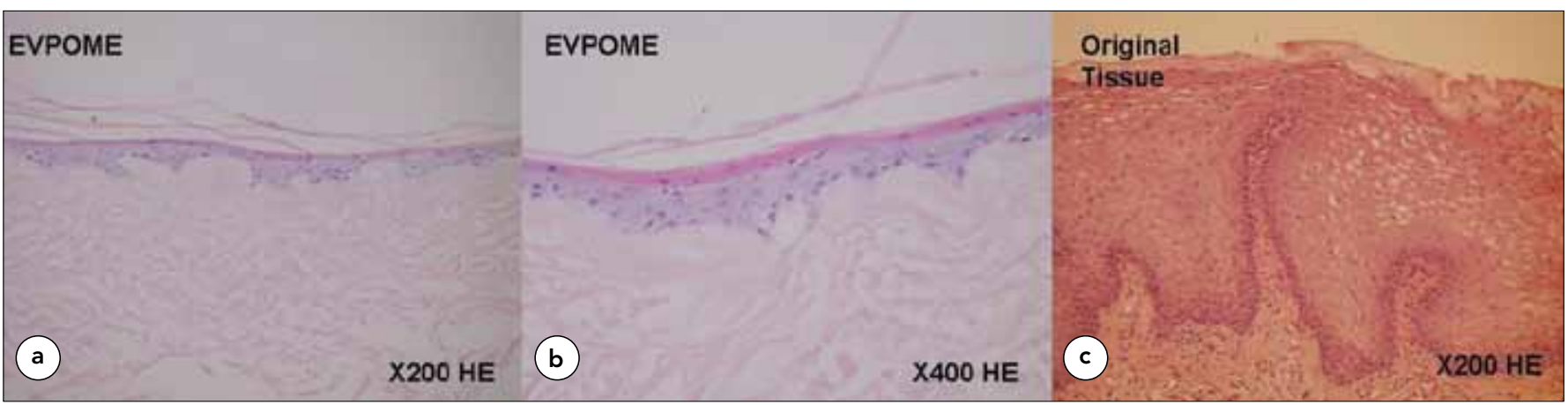

Figure 3. Histological appearances of the "EVPOME"s a) b) (x200 H.E. and x400 H.E.), and the original tissue sample c) (x200 H.E.)

"EVPOME"s showed evidence of parakeratosis. Keratinocytes of the basal layer were cuboid, and they were aligned along the "AlloDerm" surface. Under the basal layer of "EVPOME"s, "AlloDerm" showed no evidence of any cellular components and consisted of interlacing dense collagen bundles of varying sizes, indicating that the structural integrity of the extracellular matrix was intact. The thickness of the epithelial sheet was measured to be 3 to 7 cell-layer in "EVPOME" and 7 to 20 cell-layer in the original tissue sample. Despite the differences of thickness between "EVPOME" and the original tissue sample, the structure was very similar in both groups (Figure 3).

\section{Immunohistochemical evaluation}

\section{Anti-Ki-67}

The immunohistochemical comparison of the "EVPOME" and original tissue sample via $\mathrm{Ki}-67$ antibody revealed that both specimens were positive staining in their nuclei, thus confirming the existence of proliferating cells in the basal layer (Figure 4a).

\section{Anti-laminin-5}

The immunohistochemical comparison of the "EVPOME" and original tissue sample via anti-laminin- 5 antibody revealed similar linear stainings (Figure 4c).

\section{Anti-cytoceratin-3 (K-3)}

The immunohistochemical comparison of the "EVPOME" and original tissue sample via K-3 antibody revealed that both specimens were positive staining in all layers of the epithelium, except the parakeratinised layer (Figure 4b).

\section{Discussion}

The direct explant technique and the enzymatic method of obtaining cells were compared from the beginning of the respective processes. Kedjarune et al. (6) compared both the direct explant technique and the enzymatic method in cultivation of human oral keratinocytes. According to their results, the direct explant technique appeared to be more successful for culturing human oral tissue keratinocytes than the enzymatic method. The direct explant method required only small pieces of gingival tissue and generated a higher cellular product when compared with the enzymatic method. However, more time was required before subculture in the direct explant technique. These findings were in disagreement with the results of the study performed by Klingbeil et al. (7) who

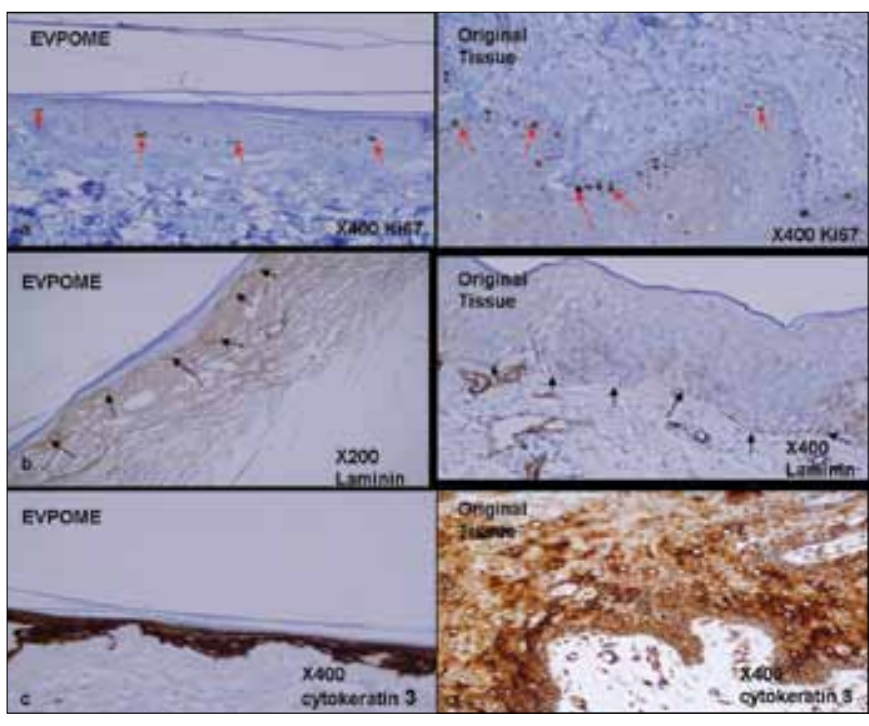

Figure 4. Immunohistochemical comparisons of the "EVPOME" and the original tissue sample by a) anti Ki-67 antibody (proliferative cells at basal layer of the epithelium are showed by red arrows), b) anti laminin-5 antibody (linear staining of the epithelium is shown by black arrows) and c) anti cytokeratin-3 antibody

stated that the enzymatic method showed the best results in the cells obtaining time needed, cell amount and life-span.

The enzymatic method appeared to require larger pieces of tissue to provide sufficient density of the seeding cells compared with the direct explant technique, however it has been suggested that the enzymatic method was faster and easier to manage (6). Freshney stated that the direct explant method should be chosen when the tissue sample is limited (22). Therefore, we think that the direct explant technique could be more suitable for intraoral grafting procedures, because the enlargement in the size of the oral tissue sample could result in more patient discomfort. Furthermore, in this study, around 8-10 pieces of tissue sample, approximately $1 \times 1 \mathrm{~mm}$ in size, were placed in the culture flasks. We think that, if the pieces of tissue sample placed in the culture flask could be more than 8-10 pieces, the average initial harvesting time for keratinocyte cultivation by the direct explant technique would be faster.

The age of the patients providing tissue samples did not appear to affect the success rate in this study. This finding was 
consistent with the results of Reid et al. (23) and Kedjarune et al. (6). However, this study contrasts with the findings of Lauer, (24) who found that age appeared to influence keratinocyte culture viability, as tissue from subjects younger than 40 years of age were cultured successfully in $80 \%$ of cases when compared to only $65 \%$ when the subjects were over 40 years of age.

It has been suggested that bacterial and fungal contamination may cause failure in both of the direct explant and the enzymatic keratinocyte culture techniques, as oral tissue is usually contaminated with a high load of microorganisms during medium preparation (6) Wanichpakorn and Kedjarune-Laggat (20) have highlighted that the contamination risk is correlated with the size of the tissue, because the tissue samples were very small and thin. The disinfection times for tissue samples can vary among different studies. In the current study, the tissue samples were not contaminated after placing in a $10 \%$ povidone iodine solution for about one minutes. In the current study, only two samples failed in keratinocyte cultivation. The reason for the failures could not be determined exactly. However, we think that these failures could depend on the harvesting, transportation and/or culturing conditions of tissue samples. In addition, no failure was detected after seeding on "Alloderm".

Technical handling involved in the direct explant method at the beginning of the process is less than the handling required for the enzymatic method. However, Daniels et al. (18) surveyed the current techniques for isolation and culture of human keratinocytes, and 21 out of 34 laboratories in England, who returned the questionnaire, reported that they used the enzymatic method, with some variation in the type, concentration and condition of the enzymes that they used, and also with some differences reported the concentration of calcium in the culture media.

The average initial harvesting time for keratinocyte cultivation by the direct explant technique was found to be14.2 days in a previous study (7). In the current study, it was $20.25 \pm 1.05$ days, somewhat longer than the previous study. Using a bigger culture plate (T-25 flask, $25 \mathrm{~cm}^{2}$ ) than this previous study may have caused this difference. According to the results of the study performed by Klingbeil et al. (7), the operating procedure used in the direct explant technique process involves fewer steps compared with the enzymatic technique. The higher success rate of direct explant technique compared with the enzymatic technique may be interpreted by the number of the steps required. In this study, the success rate of oral mucosa keratinocyte culturing by direct explant technique $(85.7 \%)$ is similar to the findings of Wanichpakorn and Kedjarune-Laggat (20) (88.9\%), higher than the study of Kedjarune et al. (6) which was about $82 \%$ and also higher than the study of Reid et al. (23), which had about $80 \%$ success rate, even though these studies used the same direct explant technique.

At the end of this study, histological findings of "EVPOME"s development showed the same epithelial architecture as the previous studies performed by Izumi et al. $(9,25)$. Also, the epithelial architecture of "EVPOME"s resembled that of normal oral mucosa. Despite the differences of thickness between "EVPOME" and original tissue sample, the structure was very similar in both groups. In addition, immunohistochemically, the existence of proliferating cells in the basal layer of "EVPOME" and similar linear stainings with laminin-5 antibody as in that of normal oral mucosa have been shown. In addition, expression of cornea-specific cytokeratin-3 in the oral epithelial cells has been shown It has also been shown by a previous animal study (26). Cytokeratin-3, reportedly, is a reliable marker for corneal differentiation and is positive for epithelial cells of the cornea, nose, and some oral mucosa, and our results are consistent with the finding about the oral mucosa (26).

\section{Conclusion}

The direct explant method used for "EVPOME" production provided successful results.from the experience of the current study, it can be concluded that the direct explant technique has two advantages in "EVPOME" production:

1. Technical handling involved in the direct explant method at the beginning of the process has fewer steps.

2. The direct explant technique could be more suitable for intraoral grafting procedures, because the enlargement in the size of the oral tissue sample could result in more patient discomfort.

The use of the direct explant technique protocol for culturing human oral mucosa keratinocyte may be more suitable for "EVPOME" production. A further study could be useful to compare histological and immunohistochemical charactheristics of "EVPOME"s produced by the direct explant technique and the enzymatic method.

\section{Acknowledgements}

Funding source: This study has been supported by "Turkish Scientific and Technological Research Council" (TUBITAK Ref. No: 110S025).

Ethical approval: This project was approved by the First Ethics Committee of Clinical Researches of Ankara, under License Number 2010/01-214.

\section{Conflict of Interest}

No conflict of interest was declared by the authors.

\section{References}

1. Alcalde J, Garcia-Tapia R, Espinosa JM, Perez N. Clinical application of free microvascular flaps in reconstructive surgery of the oral cavity. Acta Otorrinolaringol Esp 1994;45:457-60.

2. Leong DJ, Wang HL. A decision tree for soft tissue grafting. Int J Periodontics Restorative Dent 2011;31:307-13.

3. Kao SY, Lui MT, Fong J, Wu DC, Wu CH, Tu HF, et al. A method using vestibulo-sulcoplasty combining a split-thickness skin graft and a palatal keratinized mucosa graft for peri-implant tissue secondary to oral cancer surgery. J Oral Implantol 2005;31:186-91. [CrossRef]

4. Jhaveri HM, Chavan MS, Tomar GB, Deshmukh VL, Wani MR, Miller PD Jr. Acellular dermal matrix seeded with autologous gingival fibroblasts for the treatment of gingival recession: a proofof-concept study. J Periodontol 2010;81:616-25. [CrossRef]

5. Kaner D, Friedmann A. Soft tissue expansion with self-filling osmotic tissue expanders before vertical ridge augmentation: a proof of principle study. J Clin Periodontol 2011;38:95-101. [CrossRef] 
6. Kedjarune U, Pongprerachok S, Arpornmeaklong P, Ungkusonmongkhon K. Culturing primary human gingival epithelial cells: comparison of two iso lation techniques. J Craniomaxillofac Surg 2001;29:224-31. [CrossRef]

7. Klingbeil MFG, Herson MR, Cristo EB, Pinto JrDS, Yosshito D, Mathor MB. Comparison of two cellular harvesting methods for primary human oral culture of keratinocytes. Cell Tissue Bank 2009;10:197-204. [CrossRef]

8. Billingham RE, Reynolds J. Transplantation studies on sheets of pure epidermal epithelium and on epidermal cell suspensions. $\mathrm{Br}$ J Plast Surg 1952;23:25-36. [CrossRef]

9. Izumi K, Takacs G, Terashi H, Feinberg SE. Ex vivo development of a composite human oral mucosal equivalent. J Oral Maxillofac Surg 1999;57:571-7. [CrossRef]

10. Hotta T, Yokoo S, Terashi H, Komori T. Clinical and histopathological analysis of healing process of intraoral reconstruction with ex vivo produced oral mucosa equivalent. Kobe J Med Sci 2007;53:1-14.

11. Izumi K, Feinberg SE, Lida A, Yoshizawa M. Intraoral grating of an ex vivo produced oral mucosa equivalent: a preliminary report. Int J Oral Maxillofac Surg 2003;32:188-97. [CrossRef]

12. Izumi K, Song J, Feinberg SE. Development of a tissue engineered human oral mucosa: from the bench to the bed side. Cells Tissues Organs 2004;176:134-52. [CrossRef]

13. Horch RE, Debus M, Wagner G, Stark GB. Cultured human keratinocytes on type I collagen membranes to reconstitute the epidermis. Tissue Eng 2000;6:53-67. [CrossRef]

14. Schultze-Mosgau S, Lee BK, Ries J, Amann K, Wiltfang J. In vitro cultured autologous pre-confluent oral keratinocytes for experimental prefabrication of oral mucosa. Int J Oral Maxillofac Surg 2004;33:476-85. [CrossRef]

15. Lauer G, Schimming R. Tissue-engineered mucosa graft for reconstruction of the intraoral lining after freeing of the tongue: a clinical and immunohistologic study. J Oral and Maxillofacial Surg 2001;59:169-75. [CrossRef]
16. Flaxman BA, Lutzner MA, Van Scott EJ. Cell maturation and tissues organization in epithelial out-growths from skin and buccal mucosa in vitro. J Invest Dermatol 1967;49:322-32. [CrossRef]

17. Lauer G. Autografting of feeder-cell free cultured gingival epithelium. Method and clinical application. J Cranio Maxillofacial Surg 1994;22:18-22. [CrossRef]

18. Daniels JT, Kearney JN, Ingham E. Human keratinocyte isolation and cell culture: a survey of current practices in the UK. Burns 1996:22:35-9. [CrossRef]

19. Bayar GR, Aydintug YS, Gulses A, Elci P, Sarper M. A pilot study of the primary culture of the oral mucosa keratinocytes by the direct explant technique. OHDMBSC 2011;10:88-92.

20. Wanichpakorn S, Kedjarune-Laggat U. Primary cell culture from human oral tissue: gingival keratinocytes, gingival fibroblasts and periodontal ligament fibroblasts. Songklanakarin Journal of Science and Technology 2010;32:327-31.

21. Kinikoglu B, Auxenfans $C$, Pierrillas $P$, Justin V, Breton $P$, Burillon $C$, et al. Reconstruction of a full-thickness collagen- based human oral mucosa equivalent. Biomaterials 2009;30:6418-25. [CrossRef]

22. Freshney RI. Primary Culture. In: Willey-Liss Culture of animal cells: a manual of basic techniques, 5th ed.Hoboken NJ: WileyLiss, 2005: 176-97. [CrossRef]

23. Reid CBA, Cloos J, Snow GB, Braakhuis BJM. A simple and reliable technique for culturing of human oral keratinocytes and fibroblasts. Acta Otolaryngol 1997;117:628-33. [CrossRef]

24. Lauer G, Otten JE, Von Specht BU, Schilli W. Cultured gingival epithelium. A possible suitable material for pre-prosthetic surgery. J Craniomaxillofac Surg 1991;19:21-6. [CrossRef]

25. Izumi K, Feinberg SE, Terashi H, Marcelo CL. Evaluation of transplanted tissue-engineered oral mucosa equivalents in severe combined immunodeicient mice. Tissue Eng 2003;9:163-74. [CrossRef]

26. Nakamura T, Endo K, Cooper LJ, Fullwood NJ, Tanifuji N, Tsuzuki $M$, et al. The successful culture and autologous transplantation of rabbit oral mucosal epithelial cells on amniotic membrane. Invest Ophthalmol Vis Sci 2003;44:106-16. [CrossRef] 